Portland State University

PDXScholar

$5-1-2002$

\title{
Residual images in charged-coupled device detectors
}

Armin Rest

Lars Mündermann

Ralf Widenhorn

Portland State University

Erik Bodegom

Portland State University

T. C. McGlinn

Follow this and additional works at: https://pdxscholar.library.pdx.edu/phy_fac

Part of the Physics Commons

Let us know how access to this document benefits you.

\section{Citation Details}

"Residual images in charged-coupled device detectors," with R. Widenhorn, L. Mündermann, A. Rest, T.C. NcGlinn, Rev. Scient. Instr. 73, 2028 (2002)

This Article is brought to you for free and open access. It has been accepted for inclusion in Physics Faculty Publications and Presentations by an authorized administrator of PDXScholar. Please contact us if we can make this document more accessible: pdxscholar@pdx.edu. 


\section{AIP Scientific Instruments}

\section{Residual images in charged-coupled device detectors}

Armin Rest, Lars Mündermann, Ralf Widenhorn, Erik Bodegom, and T. C. McGlinn

Citation: Rev. Sci. Instrum. 73, 2028 (2002); doi: 10.1063/1.1470234

View online: http://dx.doi.org/10.1063/1.1470234

View Table of Contents: http://rsi.aip.org/resource/1/RSINAK/v73/i5

Published by the American Institute of Physics.

\section{Related Articles}

Capacitive infrared photodetector for room temperature operation Appl. Phys. Lett. 102, 103108 (2013)

UV-visible detector and LED based $\mathrm{n}-\mathrm{ZnO} / \mathrm{p}$-Si heterojunction formed by electrodeposition AIP Advances 3, 032125 (2013)

Study of sensitization process on mid-infrared uncooled PbSe photoconductive detectors leads to high detectivity J. Appl. Phys. 113, 103102 (2013)

Plasmonic enhancement of photocurrent in carbon nanotube by Au nanoparticles Appl. Phys. Lett. 102, 103102 (2013)

Operation of a titanium nitride superconducting microresonator detector in the nonlinear regime J. Appl. Phys. 113, 104501 (2013)

\section{Additional information on Rev. Sci. Instrum.}

Journal Homepage: http://rsi.aip.org

Journal Information: http://rsi.aip.org/about/about_the_journal

Top downloads: http://rsi.aip.org/features/most_downloaded

Information for Authors: http://rsi.aip.org/authors

\section{ADVERTISEMENT}
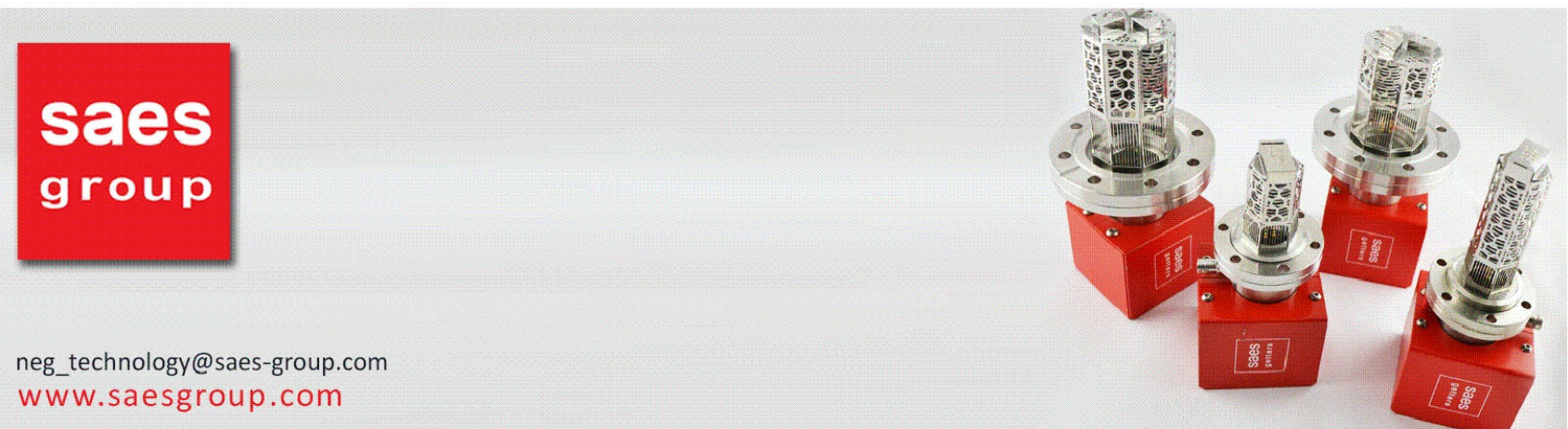


\title{
Residual images in charged-coupled device detectors
}

\author{
Armin Rest, ${ }^{\text {a) }}$ Lars Mündermann, ${ }^{\text {b) }}$ Ralf Widenhorn, Erik Bodegom, ${ }^{\text {c) }}$ and T. C. McGlinn ${ }^{\text {d) }}$ \\ Department of Physics, Portland State University, Portland, Oregon 97207-0751
}

(Received 6 November 2001; accepted for publication 25 February 2002)

We present results of a systematic study of persistent, or residual, images that occur in charged-coupled device (CCD) detectors. A phenomenological model for these residual images, also known as "ghosting," is introduced. This model relates the excess dark current in a CCD after exposure to the number of filled impurity sites which is tested for various temperatures and exposure times. We experimentally derive values for the cross section, density, and characteristic energy of the impurity sites responsible for the residual images. (C) 2002 American Institute of Physics.

[DOI: $10.1063 / 1.1470234]$

\section{INTRODUCTION}

Charge-coupled devices (CCDs) have gained widespread application in both scientific and commercial imaging. Their extreme sensitivity make them particularly adept at lowlight-level imaging, where sophisticated image processing, thermal noise, and other electronic artifacts become important.

The CCD is a device which converts incident light into photoelectrons. The photoelectrons are stored in a twodimensional (2D) array of metal-oxide-semiconductor (MOS) capacitors, or pixels, that conserve their spatial information. This electronic image is subsequently readout and recorded by sequential shifting of pixel rows toward and into a shift register, where the electrons of each pixel in a row are sequentially shifted into an analog/digital (A/D) converter and counted. This process is repeated until all pixels are counted and the image is obtained. Before an image is taken, the chip is "flushed" in order to delete all electrons that have accumulated from various processes before the image is taken. However, the raw image signal, or count, obtained is an imperfect mapping of the incident light (the true signal). In addition to the true signal, one must consider the effects of thermally generated electrons (the "dark count"), ${ }^{1-4}$ electrons generated by the bias/readout voltage (the "bias count"), the response function of the individual pixels, and optical effects such as dust shadowing or vignetting. The response function of the pixels plus optics is a multiplicative effect and can be determined by taking a "flat-field" exposure of a uniformly illuminated (flat) field. The dark count is dependent on the temperature and exposure time and can be measured for a given image by taking an equivalent exposure with the shutter closed. The bias count is introduced at each readout and can be measured by reading out a zero second exposure.

In this article, we present a systematic study of another

\footnotetext{
a) Also at Astronomy Department, University of Washington, Seattle, WA 98195.

b) Also at Department of Computer Science, University of Calgary, Calgary, Alberta T2N 1N4, Canada.

${ }^{c)}$ Electronic mail: bodegom@pdx.edu

${ }^{d)}$ Deceased, formerly at the University of Portland, Portland, OR 97203.
}

source of extraneous signal, i.e., electrons generated in previous exposures and trapped at impurity sites. These electrons are released in subsequent exposures and appear as residual images, or "ghosts." The phenomenon of "ghosting" is illustrated in Fig. 1. The image on the right is a dark frame taken shortly after a normal exposure that contained a reflected laser spot. We observe an image of the laser spot in the subsequent dark frame, residual above the normal dark count. Given a charge transfer efficiency of $>99.999 \%$, we would expect the possibility of 1 electron in 100000 to be left behind after each shift. With a maximum exposure count of less than 32000 we would thus expect no noticeable residual effects due to imperfect charge transfer. Furthermore, any effect due to imperfect charge transfer would leave a vertical streaked ghost as it sequentially transfers the charge packet from the exposure down through successive rows of pixels, something we do not observe. This ghosting phenomenon has been reported previously in the literature, but with only brief speculation as to its nature and cause. ${ }^{5}$ Epperson et al. ${ }^{6}$ previously reported the observation of such "latent" images in front-side illuminated CCDs exposed to longwavelength light, and attributed them to photoelectrons trapped at impurity sites at the epitaxy/substrate junction, which were in turn thermally released over time. Janesick and Elliott ${ }^{7}$ reported the observation of two different types of residual images: surface residual images (SRIs), which were observed when the pixels were loaded well beyond full well, and residual bulk images (RBIs), seen predominantly at longer wavelength exposure (large penetration depths). They noted that SRIs can be neutralized by appropriate voltage clocking of the CCD gates (inversion) whereas RBIs cannot.

We found that the latent images are immune to clocking voltages and appear well below full-well exposure. In accordance with previous studies, we ascribe our images to trapping sites in the "bulk," or epitaxy/substrate interface, and present here a detailed, quantitative analysis of this effect, along with an explanatory model.

\section{MEASUREMENTS AND MODEL}

For the main experiments, an AX-2 CCD camera with a Kodak KAF1600-2 sensor, manufactured by Axiom Re- 

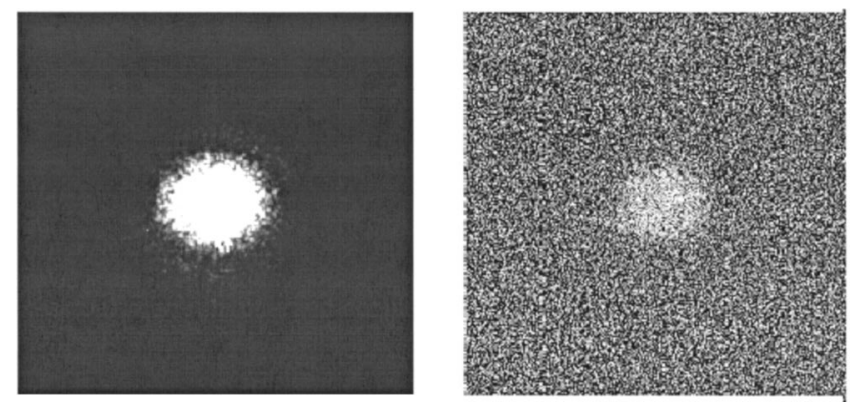

FIG. 1. Left image: Flat field of a laser spot; right image: dark image $20 \mathrm{~s}$ after the end of the flat field exposure. Note the "ghost" in the dark frame in the same part of the image where the image of the laser spot had been.

search Inc., Tucson, AZ, is used. This sensor has 1536 $\times 1024$ pixels with a pixel size of $15 \mu \mathrm{m}$ and a gain of $2 e^{-}$ per analog digital unit (ADU). In order to optimize the readout speed, a subframe of $392 \times 258$ pixels is utilized. The number of pixels is sufficiently large to do the statistical analysis. In order to verify that the residual images are not constrained to only the KAF sensor, parts of the experiments are repeated using the SBIG ST5C camera (Texas Instruments, TC-255 CCD, front side illuminated, $320 \times 240$ pixels, $10 \mu \mathrm{m}$ pixel size, and $2 e^{-}$/ADU gain). In this article, all results using the TC-255 are specifically indicated. To understand the nature of residual images, a systematic study of their time, temperature, and illumination dependence is performed. The setup for each of the following experiments to determine the number of residual electrons as a function of time at different temperatures and illumination levels is identical: First, five dark frames are taken in order to obtain the dark count without any residual counts (the "normal" dark count). Then, photoelectrons are generated at a known rate by illuminating the chip with a uniform incandescent light source well below saturation. After this flat field, a series of subsequent dark and bias frame pairs is taken. This measures the excess count per pixel above the average obtained before the flat field. Using the gain of the CCD, one derives the number of excess electrons above the average; this we denote in the remainder of this article as the number of residual electrons. The bias frame is used to account for any fluctuation in the bias. Each time series is repeated three times and an average is taken to improve the statistics.

The first measurements showed that the excess electrons (the ghost) decay exponentially over time, with a time constant which is a strong function of the temperature. One thus suspects a thermally activated source for these excess electrons. These residuals can be explained in terms of electrons photoexcited during normal exposures into midgap impurity/ interface trapping sites. The electrons trapped at the impurity/interface sites are then thermally excited into the conduction band, collected in the pixels potential wells, and appear subsequently as signal or, in our case, as residual electrons in the subsequent dark frames. This is illustrated schematically in Fig. 2.

\section{A. Populating the trapping sites}

During exposure, the traps are loaded by photoelectrons. The probability $p(n)$ that a photoelectron is trapped depends

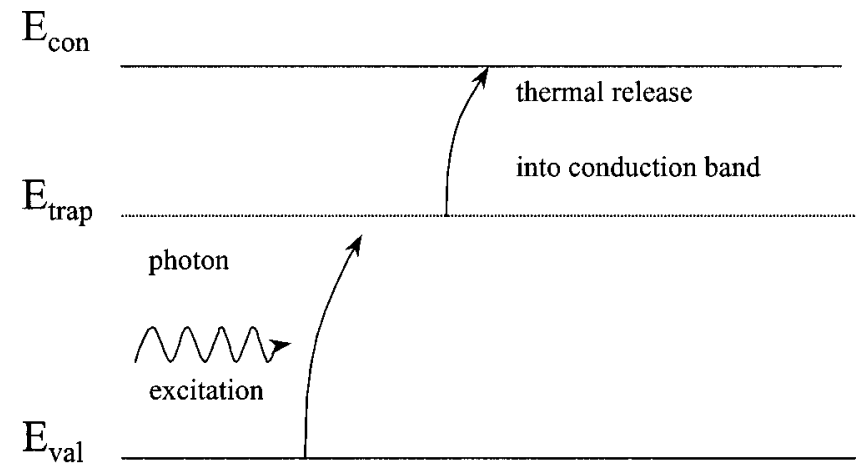

FIG. 2. Schematic diagram of photoexcited electrons moving into and out of trapping sites where $n(t)$ is the number of trapped electrons above thermal equilibrium.

on the cross section $\sigma_{0}$ of a single trapping site and on the number density of available (unoccupied) trapping sites, i.e., the number of available trapping sites per pixel. For low densities of trapping sites one expects a linear dependence of $p(n)$ on the unoccupied trapping sites and $p(n)$ can be described as

$$
p(n)=\left(n_{\max }-n\right) \sigma_{0}=p_{0}-n \sigma_{0},
$$

where $n$ is the number of filled trapping sites per pixel and $n_{\max }$ the respective total number density, i.e., $\left(n_{\max }-n\right)$ is the number of available (unoccupied) trapping sites per pixel. The probability of trapping if all traps are unloaded is then given by $p_{0}=n_{\max } \sigma_{0}$, which we denote as the initial trapping probability. During optical loading, a fraction of the trapped electrons is thermally released into the conduction band. This rate of thermal release is proportional to $n$, and we can thus write

$$
\frac{d n}{d t}=-\frac{n}{\tau},
$$

with

$$
\tau=\tau_{0} e^{\Delta E / k T},
$$

where $\tau$ is the characteristic lifetime of the trapping site and $\Delta E$ is the activation energy of the trapping site, that is, the energy of the trap below the conduction band. The change in the number of loaded traps $n$ during illumination for a given rate $r_{\text {phot }}$ of photoelectrons per pixel is then

$$
\begin{aligned}
\frac{d n}{d t}=r_{\text {phot }} p(n)-\frac{n}{\tau} & =r_{\text {phot }} p_{0}-n\left(r_{\text {phot }} \sigma_{0}+\frac{1}{\tau}\right) \\
& \equiv r_{\text {phot }} p_{0}-\frac{n}{\tau^{\prime}},
\end{aligned}
$$

with

$$
\tau^{\prime}=\left(r_{\text {phot }} \sigma_{0}+\frac{1}{\tau}\right)^{-1} .
$$

We can now obtain an expression for $n_{0}$, the number density of loaded traps after an exposure of time $t_{\text {flat }}$ :

$$
\int_{0}^{n_{0}}\left(r_{\text {phot }} p_{0}-\frac{n}{\tau^{\prime}}\right)^{-1} d n=\int_{0}^{t_{\text {flat }}} d t,
$$


which leads to

$$
n_{0}=r_{\text {phot }} p_{0} \tau^{\prime}\left(1-e^{-\left(t_{\text {flat }} / \tau\right)}\right) \text {. }
$$

For long exposures, the optical loading saturates to

$$
n_{\text {sat }}=\lim n_{0}=r_{\text {phot }} p_{0} \tau^{\prime},
$$

setting an upper limit on the maximum number of filled trapping states for a given illumination and for the temperature conditions.

\section{B. Depopulating filled trapping sites}

After an exposure is finished, electrons in the filled traps are thermally released into the conduction band. Using Eq. (2) and applying the boundary condition that $n_{0}$ trapping sites are populated at time $t=0$ after the illumination has ended, one can express the subsequent thermal release and the evolution of trapped sites as

$$
\begin{aligned}
& n=n_{0} e^{-t / \tau}, \\
& \frac{d n}{d t}=-\frac{n_{0}}{\tau} e^{-t / \tau} .
\end{aligned}
$$

In order to probe this exponential release, dark frames are taken subsequent to the illumination in the manner described above. The residual electrons $N_{d}\left(t_{0}\right)$ accumulated in a pixel during the dark integration with an exposure time of $t_{d}$ taken at time $t_{0}$ after the light exposure has ended can be expressed as

$$
N_{d}\left(t_{0}\right)=\int_{t_{0}}^{t_{0}+t_{d}}\left(-\frac{d n}{d t}\right) d t
$$

Since it is essential to have a good time resolution of data points, especially at high temperatures when the time constants are short, we take short dark exposures of length $t_{d}=6 \mathrm{~s}$. However, using such short exposures poses another problem. There are significant contributions to the number of residual electrons that accumulate in a pixel during the preceding flushing (i.e., clearing) of the CCD and the subsequent readout process. The measured flushing and readout times for the subframe are $t_{f}=1.1 \mathrm{~s}$ and $t_{r}=3.75 \mathrm{~s}$, which are of the same order as the actual dark exposure time. We explain this effect in the following example of how $R$ pixel rows are read out: The first row readout contains only residual electrons from the preceding dark integration. Until the $r$ th row is read out, however $\left(t_{r} r / R\right)$ seconds pass, and in this time additional residual electrons are accumulated in this row. This means each pixel accumulates additional residual electrons during readout, the number of which differs from row to row. In order to simplify, we can calculate the number of residual electrons accumulated on average during readout in a pixel as

$$
N_{r}\left(t_{0}\right)=\int_{t_{0}+t_{d}}^{t_{0}+t_{d}+t_{r}}\left(1-\frac{t-\left(t_{0}+t_{d}\right)}{t_{r}}\right)\left(-\frac{d n}{d t}\right) d t .
$$

In a similar manner, the average number of residual electrons accumulated in a pixel during the flushing process is

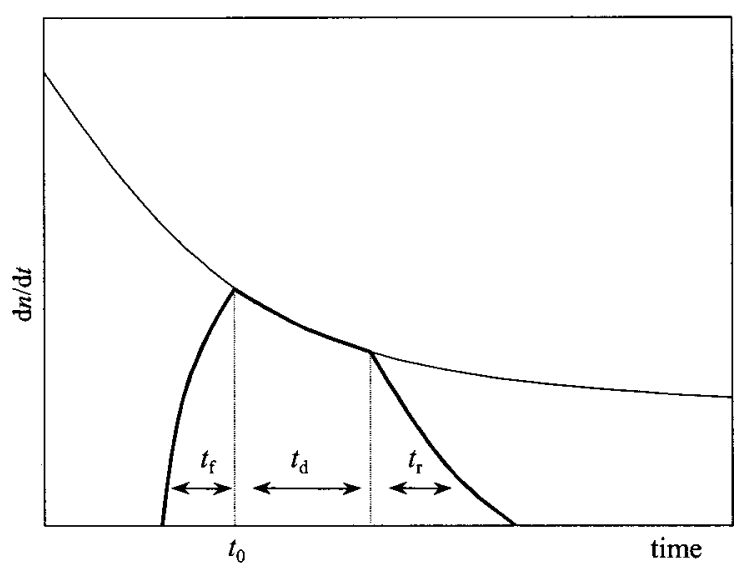

FIG. 3. Illustration of the collection of residual electrons during the flushing, dark exposure, and readout processes. The actual dark exposure starts at $t_{0}$, and lasts for time $t_{d}$. The amount of residual electrons per pixel $N\left(t_{0}\right)$ measured is given by the area under the thick line. The thin line indicates the rate $d n / d t$ of thermally released electrons by the trapping sites.

$$
N_{f}\left(t_{0}\right)=\int_{t_{0}-t_{f}}^{t_{0}} \frac{t-\left(t_{0}-t_{f}\right)}{t_{f}}\left(-\frac{d n}{d t}\right) d t .
$$

The total number of residual electrons per pixel (averaged over the whole subframe), $N\left(t_{0}\right)$, generated by electrons released from trapping sites during flushing, dark integration, and readout, and collected in a dark exposure taken at time $t_{0}$ after the flat field (which populated the trapping sites) is then given by

$$
N\left(t_{0}\right)=N_{f}\left(t_{0}\right)+N_{d}\left(t_{0}\right)+N_{r}\left(t_{0}\right) .
$$

Substituting, integrating, and simplifying leads to

$$
\begin{aligned}
N\left(t_{0}\right)= & n_{0} e^{-t_{0} / \tau}\left\{\frac{1}{t_{f}}\left[\tau\left(e^{t_{f} / \tau}-1\right)-t_{f}\right]+\left(1-e^{-t_{d} / \tau}\right)\right. \\
& \left.+\frac{e^{-t_{d} / \tau}}{t_{r}}\left[\tau\left(e^{-t_{r} / \tau}-1\right)+t_{r}\right]\right\} .
\end{aligned}
$$

Note that $N_{d}\left(t_{0}\right)$ is not the total number of electrons in a given pixel, but, rather, the average number of residual or excess electrons per pixel. This is due to depopulating of the trapping sites compared to what is measured in a normal dark frame. Equation (8) has only two free parameters, the characteristic time $\tau$ and the initial number density of loaded traps $n_{0}$. The accumulation of residual electrons is schematically displayed in Fig. 3. The quantity of residual electrons per pixel $N\left(t_{0}\right)$ measured is given by the area under the thick line. The thin line indicates the rate $d n / d t$ of thermally released electrons per pixel by the trapping sites.

\section{RESULTS AND DISCUSSION}

In order to test the dependence of the number of residual electrons on the temperature, time series for several temperatures are obtained in the manner described previously and fitted to Eq. (8). As one can see in the upper panel of Fig. 4, the agreement between the model and data is excellent. Applying Eq. (3) to the temperature dependence of $\tau$ (see the open symbols and solid line in Fig. 5) yields 

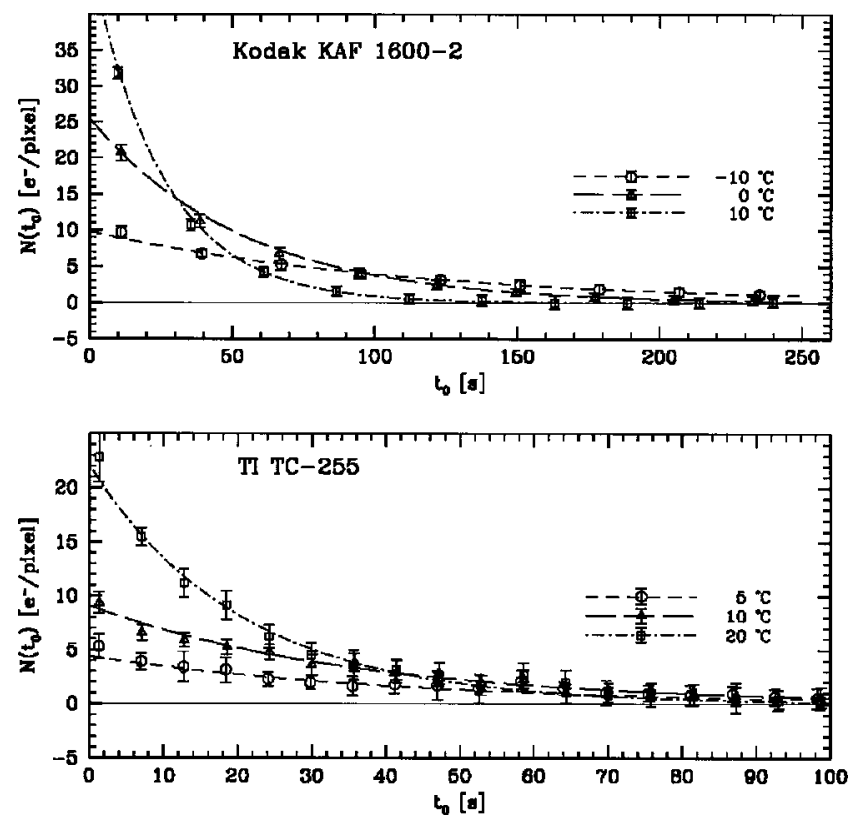

FIG. 4. Residual electrons for a sequence of dark images taken at time $t_{0}$ after a flat field exposure at several temperatures with the Kodak KAF 1600-2 Sensor (upper panel) and with the TI TC- 255 Camera (lower panel). The lines are the best fit of the data to Eq. (8).

$$
\begin{aligned}
& \Delta E=0.48 \pm 0.02 \mathrm{eV}, \\
& \tau_{0}=7.1 \times 10^{-8} \pm 4.9 \times 10^{-8} \mathrm{~s} .
\end{aligned}
$$

By assuming a silicon band gap of $1.14 \mathrm{eV}$ at ambient temperature, one obtains $0.66 \mathrm{eV}$ for the impurity energy $E_{\text {trap }}$ above the valence band. In order to test whether the residuals are a more widely distributed artifact of CCDs, we performed an experiment with virtually the same setup using the SBIG ST5C camera (Texas Instruments, TC-255 CCD), which shows the same effect (see lower panel of Fig. 4). Utilizing the same analysis as described above, we find the impurity energy $E_{\text {trap }}$ of the TC- 255 to be $0.75 \pm 0.06 \mathrm{eV}$ (see the closed squares and dashed line in Fig. 5). The midgap impurity levels correspond well with values of activation energies determined by dark current measurements for "hot"

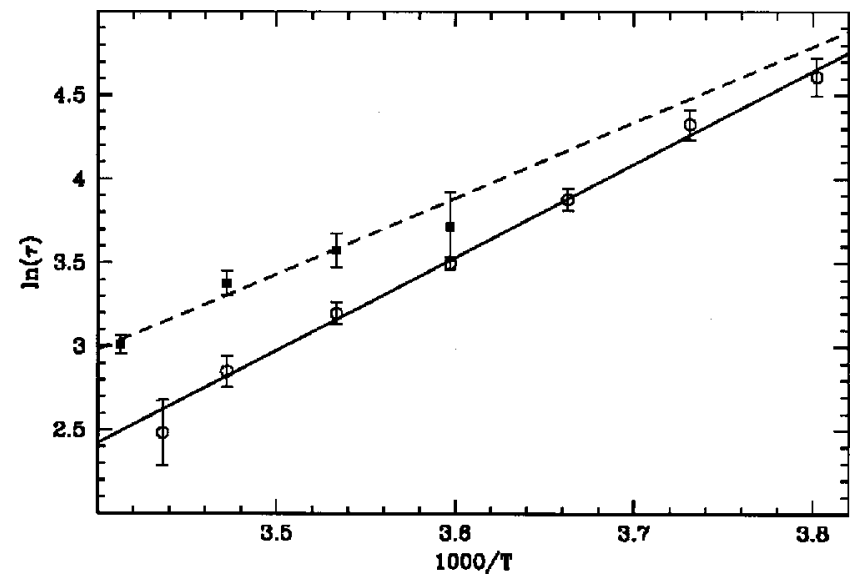

FIG. 5. Characteristic lifetime vs the inverse temperature for the Kodak KAF 1600-2 (open circles and solid line) and the TI TC-255 (closed squares and dashed line). The slope of the fitted line gives the characteristic energy $\Delta E$.

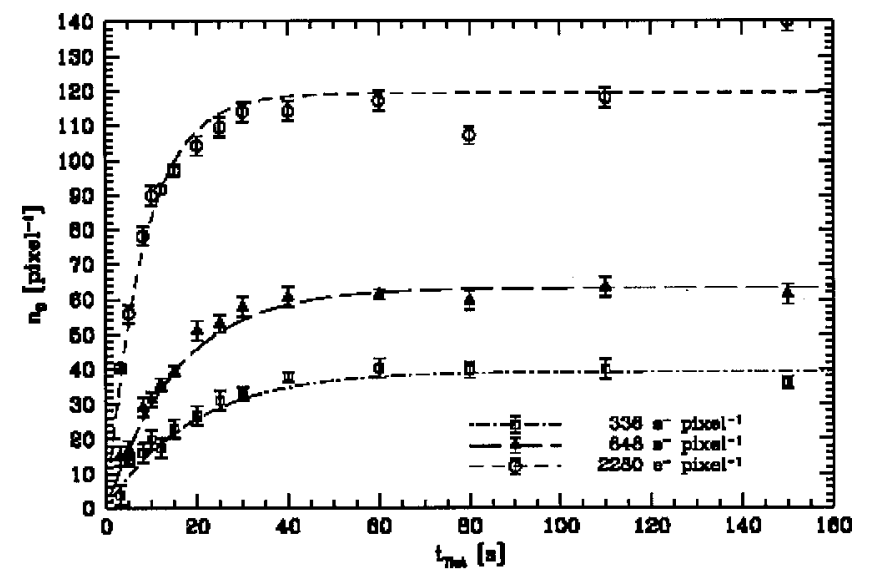

FIG. 6. Number density $n_{0}$ of filled trapping sites after a flat field exposure of illumination levels that generate 336,648 , and 2280 photoelectrons per second at $10{ }^{\circ} \mathrm{C}$. The lines are the fit of Eq. (4) to the data.

pixels (i.e., pixels with a high dark current due to a large number of impurities) $)^{1,8,9}$ and can be associated with $\mathrm{Au}, \mathrm{Ni}$, or $\mathrm{Co}^{3}$

The number of filled trapping sites $n_{0}$ after a flat field (or any other light exposure) ends depends on $\tau$ (i.e., on the temperature $T$ ), on the rate of photoelectrons generated $r_{\text {phot }}$, and on the exposure time $t_{\text {flat }}$ [see Eq. (4)]. In order to test these dependencies, the time series are repeated for various temperatures $\left(0,5\right.$, and $\left.10^{\circ} \mathrm{C}\right)$, exposure times $(3,5,8,10$, $12,15,20,25,30,40,60,80,110$, and $150 \mathrm{~s}$ ), and illumination conditions $\left(336,648\right.$, and $\left.2280 e^{-} / \mathrm{s}\right)$ and fitted to Eq. (8). This yields values for $n_{0}\left(T, r_{\text {phot }}, t_{\text {flat }}\right)$ which are then subsequently fitted to Eq. (4). As one can see in Figs. 6 and 7 the experimental data are in excellent agreement with the model of the population of trapping sites. We find the following detector-specific values for $\sigma_{0}$, the cross section of a single trapping site, and $n_{\max }$, the total number of trapping sites per pixel:

$$
\begin{aligned}
& \sigma_{0}=7.71 \times 10^{-3} \pm 2.8 \times 10^{-4} \mu \mathrm{m}^{2} ; \\
& n_{\max }=184.0 \pm 4.2 \mathrm{pixel}^{-1}=0.818 \pm 0.019 \mu \mathrm{m}^{-2} .
\end{aligned}
$$

The initial trapping probability $p_{0}$ [see Eq. (1)] is then

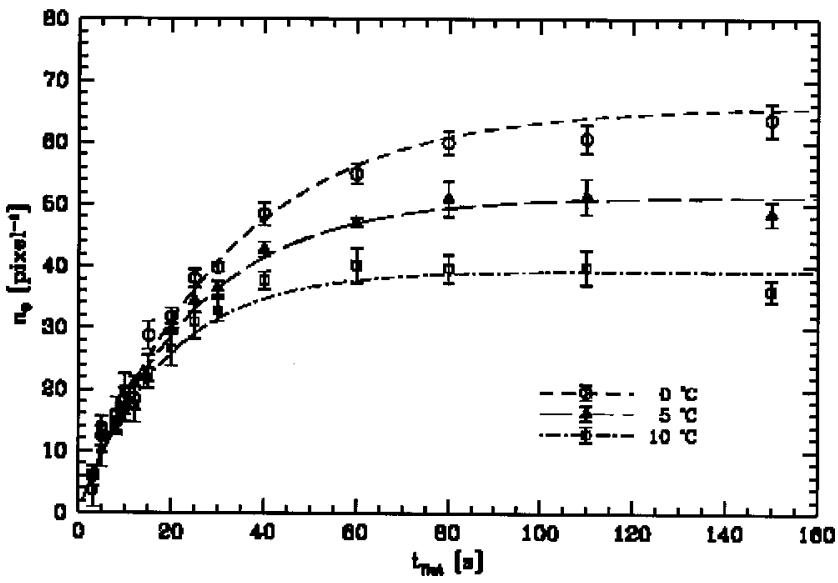

FIG. 7. Number density $n_{0}$ of filled trapping sites after a flat field exposure at three different temperatures $\left(0,5\right.$, and $\left.10^{\circ} \mathrm{C}\right)$ for a constant illumination level of 336 photoelectrons per second. 


$$
p_{0}=\sigma_{0} n_{\max }=6.3 \times 10^{-3} \pm 2.7 \times 10^{-4} .
$$

This means that close to $1 \%$ of the photoelectrons are trapped at the time all trapping sites are unoccupied. Figure 6 shows, as an example, the dependence of $n_{0}$ on various illumination conditions at $10^{\circ} \mathrm{C}$ and the respective fits. One notes immediately that the initial loading of sites is, as expected, linearly dependent on the level of illumination. For longer exposure times $t_{\text {flat }}$, however, the loading saturates to $n_{\text {sat }}$ and is basically independent of the exposure time $t_{\text {flat }}$ [see Eq. (5)]. For a constant level of illumination, the initial loading of the sites is the same for different temperatures (see Fig. 7). However, at lower temperatures thermal release of trapped electrons is slower than that at high temperatures, and therefore the trapping sites are filled to a higher level before equilibrium between release and loading is reached.

We performed some experiments on a back-side illuminated CCD detector which showed only a small residual signal. This is reasonable since the bulk substrate is thinned and thus fewer trapping sites are available. Also, at very low temperatures, routinely used for many CCDs, the characteristic lifetime of the states is long and therefore the trapped electrons are released over a time much longer than the typi- cal integration time of an image. Nevertheless, for detectors operating at medium low temperatures, residuals can appear in the images. For example, a saturated star in an astronomical CCD image can leave a measurable residual in a subsequent image with a long exposure time. The analysis can also be used by manufacturers to gain information and better understanding of the nature and density of impurity sites in CCD detectors.

${ }^{1}$ R. D. McGraph, J. Doty, G. Lupino, G. Ricker, and J. Vallerga, IEEE Trans. Electron Devices ED-34, 2555 (1987).

${ }^{2}$ W. J. Toren and J. Bisschop, Philips J. Res. 48, 207 (1994).

${ }^{3}$ W. C. McColgin, J. P. Lavine, J. Kyan, D. N. Nichols, and C. V. Stancampiano, Int. Electron Devices Meet. 92, 113 (1992).

${ }^{4}$ N. S. Saks, IEEE Electron Device Lett. EDL-1, 131 (1980).

${ }^{5}$ J. Janesick, Scientific Charge-Coupled Devices (SPIE, Bellingham, 2001).

${ }^{6}$ P. Epperson, J. V. Sweedler, M. B. Denton, G. R. Sims, T. W. McCurnin, and R. S. Aikens, Opt. Eng. (Bellingham) 26, 721 (1987).

${ }^{7}$ J. Janesick and T. Elliott, ASP Conf. Ser. 23, 1 (1992).

${ }^{8}$ L. Mündermann, M.S. thesis, Portland State University, Portland, OR, 1998.

${ }^{9}$ R. Widenhorn, A. Rest, L. Mündermann, and E. Bodegom, J. Appl. Phys. 89, 8179 (2001). 